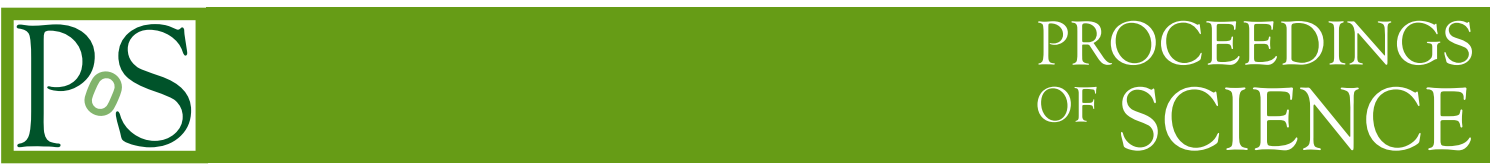

\title{
Hard X-ray Variability of the Brightest Swift/BAT AGN
}

\author{
Claudio Ricci* \\ ISDC Data Centre for Astrophysics \\ Geneva Observatory, University of Geneva \\ E-mail: claudio.ricci@unige.ch \\ S. Paltani \\ ISDC Data Centre for Astrophysics \\ Geneva Observatory, University of Geneva
}

\section{S. Soldi}

Laboratoire AIM - CNRS - CEA/DSM - Université Paris Diderot (UMR 7158), CEA Saclay, DSM/IRFU/SAp, F91191 Gif-sur-Yvette, France

\section{T. J.-L. Courvoisier}

ISDC Data Centre for Astrophysics

Geneva Observatory, University of Geneva

\begin{abstract}
Variability is one of the hallmarks of Active Galactic Nuclei. The Burst Alert Telescope onboard of Swift, with its homogeneous coverage of the sky is a formidable tool to study variability at hard X-rays. We present here the analysis of the 1-month binned Swift/BAT lightcurves of the 20 brightest Active Galactic Nuclei in the hard X-ray sky. The sample consists of 2 blazars, 3 radio galaxies, 6 Seyfert 1/1.5s, 8 Seyfert 2s and 1 Narrow Line Seyfert 1. We found that all the objects show variability, and most of them have a value of the fractional root mean squared variability amplitude of $F_{\mathrm{var}} \sim 0.2-0.3$. We did not find any significant correlation of $F_{\mathrm{var}}$ with the column density or the luminosity in our small sample.
\end{abstract}

Fast $X$-ray timing and spectroscopy at extreme count rates

February 7-11, 2011

Champéry, Switzerland

* Speaker. 


\section{Introduction}

Active Galactic Nuclei (AGN) are amongst the most luminous X-ray sources in the sky. AGN are thought to be powered by accretion onto supermassive black holes [1], with their X-ray emission probably originating in a hot corona sandwiching the accretion disk [2] in radio-quiet objects, and in the jet in radio-loud AGN (e.g., [3]). Variability is one of the key features of AGN, and it was found to be significative in the X-ray band already in early observations of nearby Seyfert galaxies (Sy) performed by Ariel V [4]. The X-ray variability of AGN is aperiodic, and their power spectral density distribution (PSD) can be normally described with a broken power law, with indices ranging between -1 and -2 [5].

The Burst Alert Telescope (BAT) onboard of Swift [6] scans continuously the whole sky in the 14-195 keV energy range, and is thus an extremely well suited instrument for studying AGN variability at hard X-rays. Here we report a study of the hard X-ray variability of a small sample of AGN. The sample consists of the 20 brightest AGN detected by Swift/BAT, of these 2 are blazars, 2 narrow-line radio galaxies (NLRG), 1 broad-line radio galaxy (BLRG), 6 Seyfert 1/1.5s, 8 Seyfert 2 s and 1 Narrow Line Seyfert 1 (NLS1). The 1-month binned light curves have been taken from the NASA Swift/BAT 58 months catalog ${ }^{1}$ [7].

\section{Variability estimators}

A way to estimate the variability is through the fractional root mean squared (rms) variability amplitude $F_{\text {var }}([8])$, defined as

$$
F_{\mathrm{var}}=\sqrt{\frac{S^{2}-\overline{\sigma_{e r r}^{2}}}{\bar{x}^{2}}}
$$

Where the sample variance $S^{2}$ is given by

$$
S^{2}=\frac{1}{N-1} \sum_{i=1}^{N}\left(x_{i}-\bar{x}\right)^{2},
$$

while the mean square error $\overline{\sigma_{e r r}^{2}}$ by

$$
\overline{\sigma_{e r r}^{2}}=\frac{1}{N} \sum_{i=1}^{N} \sigma_{e r r, i}^{2}
$$

The error of $F_{\mathrm{var}}$ is given by

$$
\operatorname{err}\left(F_{\mathrm{var}}\right)=\sqrt{\left(\sqrt{\frac{1}{2 N}} \cdot \frac{\overline{\sigma_{e r r}^{2}}}{\bar{x}^{2} F_{\mathrm{var}}}\right)^{2}+\left(\sqrt{\frac{\overline{\sigma_{e r r}^{2}}}{N}} \cdot \frac{1}{\bar{x}}\right)^{2}}
$$

In the following we will use $F_{\text {var }}$ to characterize the variability of the objects in our sample.

\footnotetext{
${ }^{1}$ http://swift.gsfc.nasa.gov/docs/swift/results/bs58mon/
} 
Table 1: Properties of the sources of our sample: (1) detection significances, (2) luminosities in the 14$195 \mathrm{keV}$ energy range, (3) fractional rms variability amplitudes on a timescale of 30 days, (4) hydrogen column densities.

\begin{tabular}{|c|c|c|c|c|c|}
\hline Source & $\begin{array}{c}(1) \\
\text { Det. Significance } \\
{[\sigma]}\end{array}$ & $\begin{array}{c}(2) \\
\log L_{14-195 \mathrm{keV}} \\
{\left[\mathrm{erg} \mathrm{s}^{-1}\right]}\end{array}$ & $\begin{array}{c}(3) \\
F_{\text {var }}(30 \text {-days })\end{array}$ & $\begin{array}{c}(4) \\
N_{\mathrm{H}} \\
{\left[\mathrm{cm}^{-2}\right]}\end{array}$ & Type \\
\hline Cen A & 428.7 & 44.01 & $0.399 \pm 0.002$ & $12^{a}$ & NLRG \\
\hline NGC 4151 & 275.0 & 44.11 & $0.280 \pm 0.004$ & $6.9^{b}$ & Sy 1.5 \\
\hline $3 \mathrm{C} 273$ & 156.8 & 47.47 & $0.31 \pm 0.01$ & $0.5^{b}$ & Blazar \\
\hline NGC 4388 & 110.7 & 44.64 & $0.31 \pm 0.01$ & $27^{b}$ & Sy 2 \\
\hline Mrk 421 & 109.5 & 45.46 & $0.96 \pm 0.01$ & $0.1^{b}$ & Blazar \\
\hline Circinus Galaxy & 101.7 & 43.09 & $0.13 \pm 0.01$ & $360^{b}$ & Sy 2 \\
\hline IC 4329A & 101.1 & 45.22 & $0.19 \pm 0.02$ & $0.4^{b}$ & Sy 1 \\
\hline NGC 2110 & 98.1 & 44.60 & $0.32 \pm 0.01$ & $4.3^{c}$ & Sy2 \\
\hline NGC 5506 & 95.0 & 44.31 & $0.27 \pm 0.02$ & $3.4^{c}$ & NLS1 \\
\hline MCG $-05-23-016$ & 90.4 & 44.50 & $0.21 \pm 0.01$ & $1.6^{c}$ & Sy2 \\
\hline IGR J21247+5058 & 83.3 & 45.25 & $0.31 \pm 0.01$ & $0.6^{b}$ & BLRG \\
\hline NGC 4945 & 76.1 & 43.37 & $0.35 \pm 0.02$ & $400^{b}$ & Sy2 \\
\hline Mrk 348 & 70.4 & 44.91 & $0.28 \pm 0.02$ & $30^{c}$ & Sy2 \\
\hline NGC 3783 & 68.7 & 44.60 & $0.26 \pm 0.02$ & $0.1^{c}$ & Sy1.5 \\
\hline NGC 4507 & 64.6 & 44.77 & $0.29 \pm 0.02$ & $29^{c}$ & Sy 2 \\
\hline NGC 3516 & 62.3 & 44.33 & $0.30 \pm 0.02$ & $4^{c}$ & Sy 1.5 \\
\hline NGC 7172 & 60.1 & 44.46 & $0.35 \pm 0.04^{*}$ & $9^{c}$ & Sy 2 \\
\hline NGC 3227 & 56.2 & 43.57 & $0.16 \pm 0.21$ & $6.8^{c}$ & Sy 1.5 \\
\hline Cyg A & 54.0 & 46.01 & $0.34 \pm 0.02$ & $11^{b}$ & NLRG \\
\hline $\mathrm{MCG}+08-11-011$ & 49.0 & 45.09 & $0.35 \pm 0.03$ & $0.2^{c}$ & Sy 1.5 \\
\hline Crab & 7496 & - & $0.0215 \pm 0.0004$ & - & - \\
\hline
\end{tabular}

Notes. ${ }^{a}[9],{ }^{b}[10]$ and references therein, ${ }^{c}$ [11] and ref. therein. $*$ "Flare" of January 2008 removed.

\section{Results}

In Table 1 are listed the values of the fractional rms variability amplitude of the objects of our sample, and as a check, the value obtained from the light curve of the $\mathrm{Crab}\left(F_{\mathrm{var}} \sim 0.02\right)$. The value of the Crab can be associated to the systematic error of the Swift/BAT data. In Fig. 1, we show the light curves of 6 out of the 20 sources of our sample. All the sources of our sample show hard X-ray variability on the time-scale of one month. As it can also be seen from Fig. 2, the value of the fractional rms variability amplitude is $F_{\text {var }} \sim 0.2-0.3$ for most of the objects of the sample, with the average value being $\overline{F_{\text {var }}}=0.32$. The blazar Mrk 421 shows a much stronger variability $\left(F_{\text {var }} \sim 0.96\right)$ than the average value of the sample. Amongst the radio-quiet NGC 7172 is the most variable, with a value of $F_{\text {var }} \sim 0.48$. This is due to what would appear to be a flare, registered in January 2008. Excluding this outlier point NGC 7172 shows a variability consistent with the 

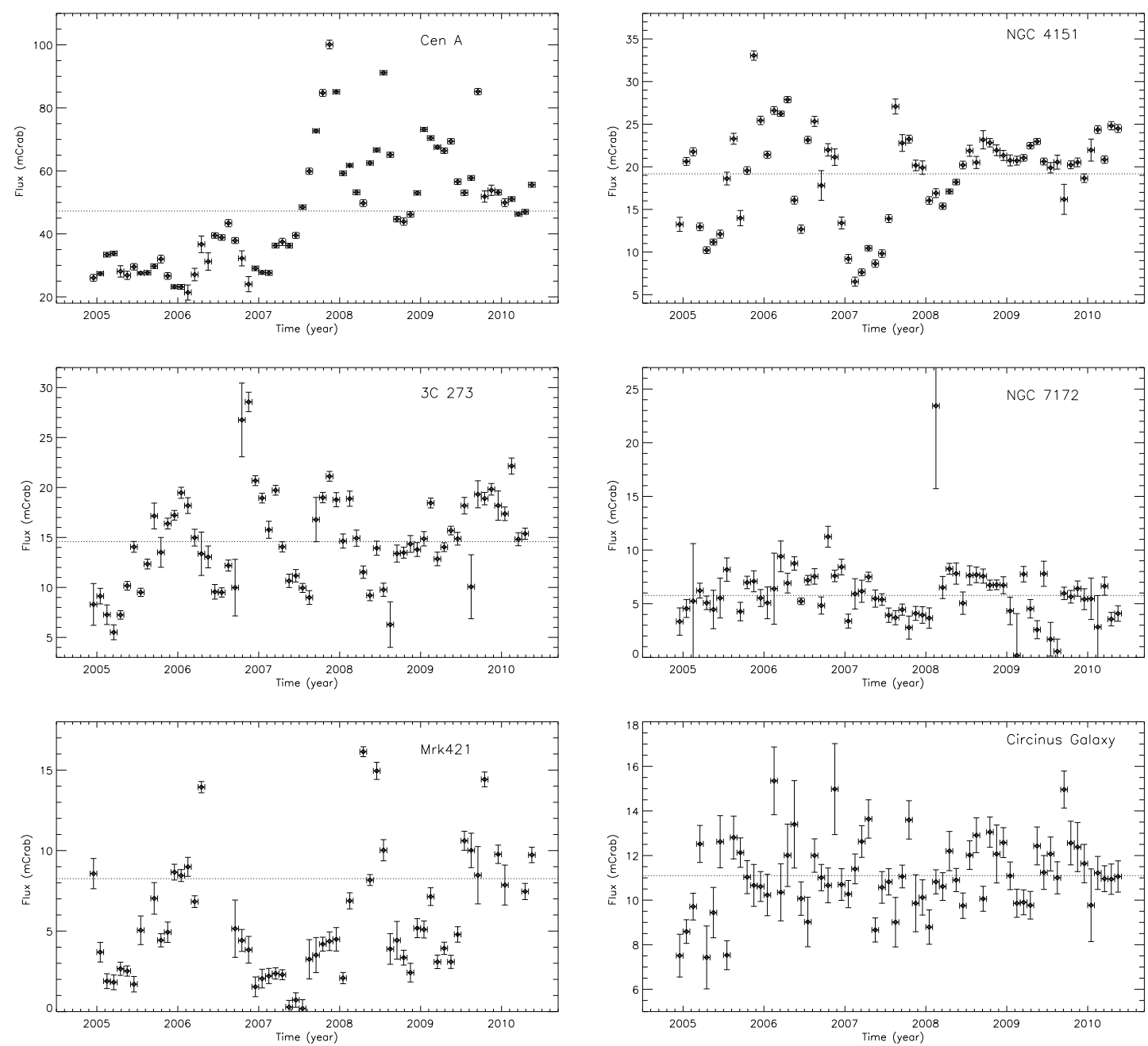

Figure 1: Swift/BAT 30-days binned light curves in the 14-195 keV band. The dotted horizontal lines represent the average value for each object.

average of our sample $\left(F_{\mathrm{var}}=0.35 \pm 0.04\right)$. At the other end of the distribution, the Compton-thick Seyfert 2 Circinus Galaxy and the Seyfert 1 IC 4329A show the smallest amounts of variability ( $F_{\text {var }} \sim 0.13$ and $F_{\text {var }} \sim 0.19$, respectively). The low value of $F_{\text {var }}$ of Circinus Galaxy is very likely related to the reflection-dominated nature of its hard X-ray spectrum.

\section{Variability vs Luminosity and Column density}

An inverse correlation between the variability amplitude in the X-rays and the X-ray luminosity of AGN was found by [12] using EXOSAT data. More recently, [13] studied the hard X-ray variability of the 44 brightest AGN detected by BAT after 9 months of operations, and found that possibly this anti-correlation is extended to the hard X-ray band (see also [14]). They also found a possible correlation between the hydrogen column density $N_{\mathrm{H}}$ and the variability amplitude. We investigated the existence of these two correlations in our sample (see Figs. 3 and 4). The variability amplitudes in our sample are confined in a small range of values, and no correlation with other 


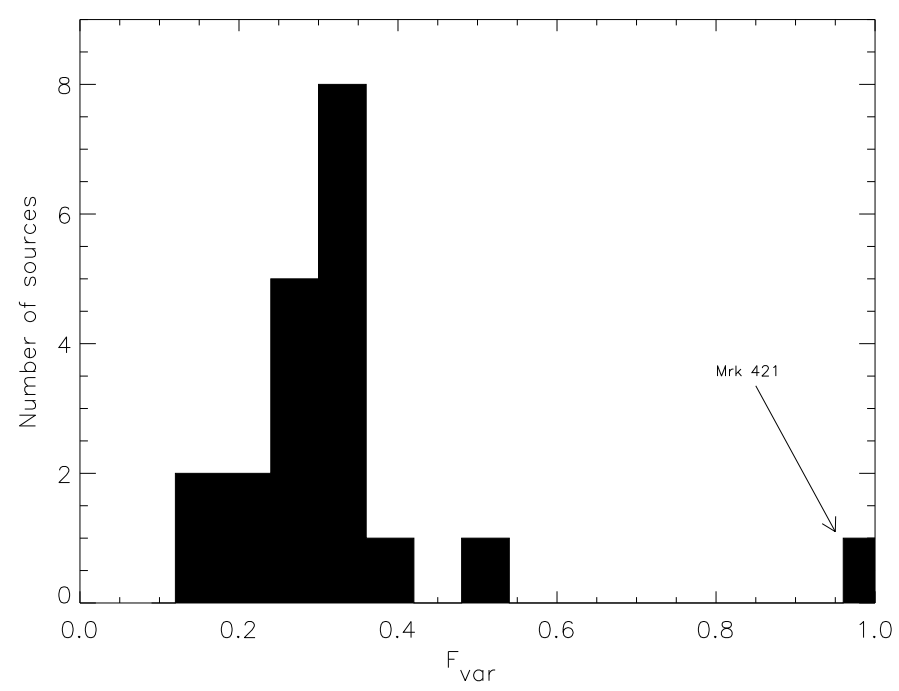

Figure 2: Distribution of the fractional rms variability amplitude for the 20 sources of our sample.

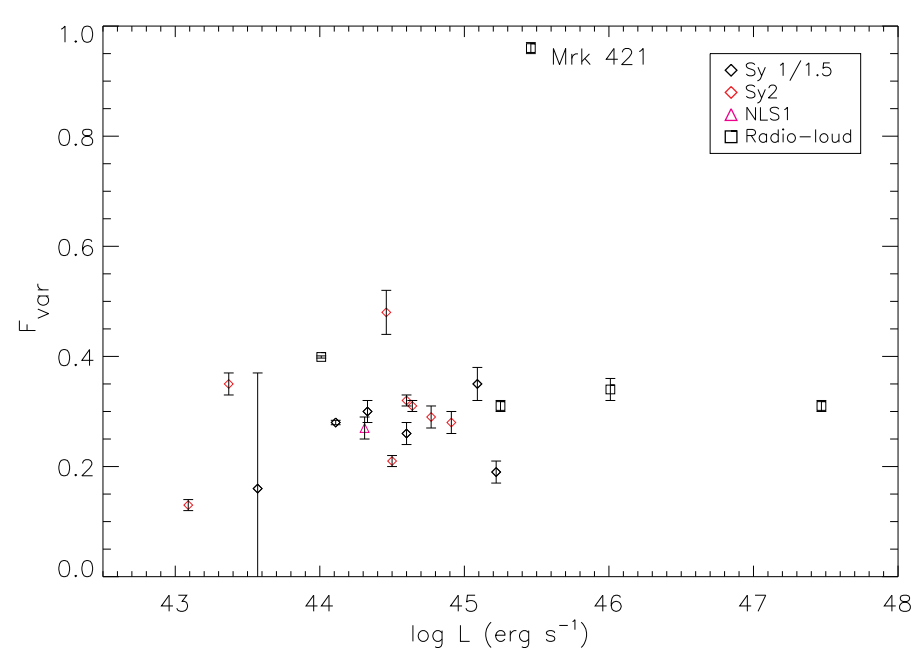

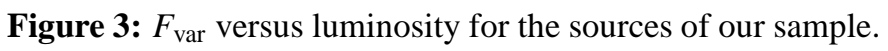

parameters is evident. A Spearman rank test gives a correlation coefficient between $F_{\text {var }}$ and the luminosity of $r_{s}=0.27$, while it is $r_{s}=0$ between $F_{\mathrm{var}}$ and $N_{\mathrm{H}}$. These values correspond to a probability of correlation of $78 \%$ in the first case, and of $0 \%$ in the second case. Similar results are obtained also considering Mrk 421 as an outlier. No significant correlation is found also dividing the sample in three categories (Sy 1/1.5, Sy2, radio-loud). The lack of correlations might be due to the limited sample we used, and further studies are needed to better probe it.

\section{Conclusions}

Studying the 1-month binned Swift/BAT light-curves of the 20 brightest objects after 58 months 


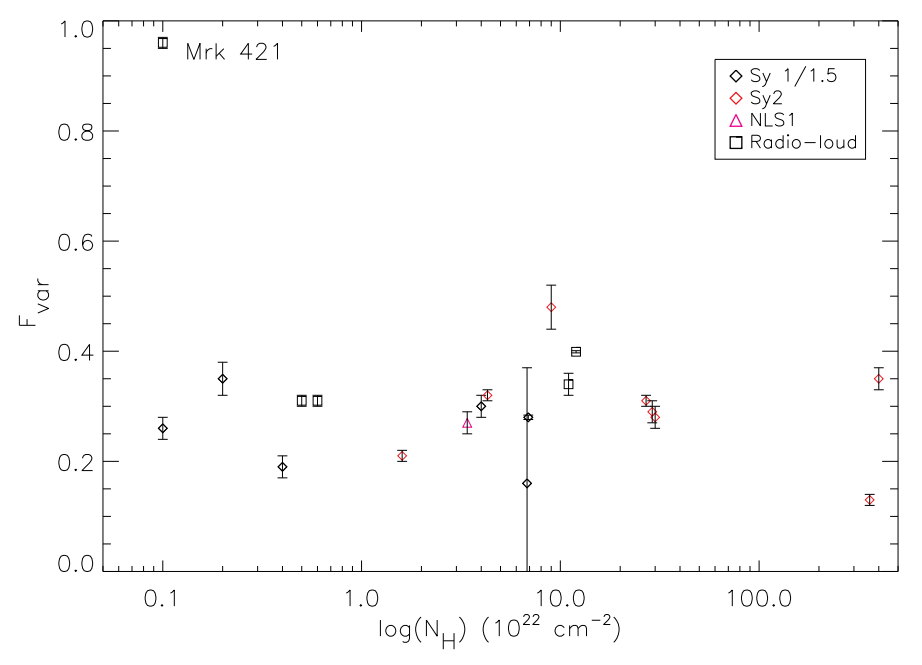

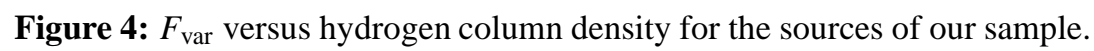

of observations, we found that all the objects in our sample show variability, ranging between $F_{\text {var }}=0.13$ and $F_{\text {var }}=0.96$, for Circinus galaxy and Mrk 421, respectively. The average value of the variability amplitude if $F_{\mathrm{var}} \sim 0.3$. We did not find any significant correlation of the variability amplitude with the luminosity and the hydrogen column density.

\section{References}

[1] Rees, M. J. 1984, ARA\&A 22, 471

[2] Haardt, F., \& Maraschi, L. 1991, ApJ 380, L51

[3] Boettcher, M., arXiv:1006.5048

[4] Marshall, N., Warwick, R. S., Pounds, K. A. 1981, MNRAS, 194, 987

[5] McHardy, I. M., \& Czerny, B. 1987, Nature, 325, 696

[6] Barthelmy, S. D., Barbier, L. M., Cummings, J. R., et al. 2005, SSRv, 120, 143

[7] Baumgartner et al, 2011 ApJS, submitted

[8] Edelson, R. A.; Krolik, J. H.; Pike, G. F. 1990, ApJ 359, 86

[9] Beckmann, V.; Jean, P.; Lubinski, P., et al. 2011, A\&A in press, arXiv:1104.4253v2

[10] Beckmann, V.; Soldi, S.; Ricci, C., et al. 2009, A\&A 505, 417

[11] Ricci, C.; Walter, R.; Courvoisier, T. J.-L.; et al. 2011, A\&A in press, arXiv:1104.3676

[12] Barr, P., \& Mushotzky, R. F. 1986, Nature, 320, 421

[13] Beckmann, V.; Barthelmy, S. D.; Courvoisier, T. J.-L.; et al. 2007, A\&A 475, 827

[14] Soldi, S.; Ponti, G.; Beckmann, V.; et al. 2010, arXiv1001.4348S 